\title{
Klebstoffindustrie weiter auf Wachstumskurs
}

Mit einer produzierten Menge von 870.000 Tonnen hat die deutsche Klebstoffindustrie im Jahr 2011 zum zweiten Mal in Folge eine Rekordmarke erreicht und damit ihren europäischen Marktanteil auf über 36 Prozent ausgebaut. Für 2012 erwartet die Industrie ein organisches Wachstum ihres Gesamtbranchenumsatzes von ca. 3,5 Prozent auf etwa 3,4 Milliarden Euro. Bis Ende 2012 werden über 1,4 Millionen Tonnen Kleb-, Dicht- und zementäre Bauklebstoffe sowie fast eine Milliarde Quadratmeter Klebebänder und -folien produziert.

$\mathrm{D}$ e Nachfrage nach Klebstoffen entwickelt sich weiterhin positiv. In einer aktuellen Repräsentativ-Umfrage des Industrieverband Klebstoffe e.V. (IVK) bestätigen die dem Branchenverband angeschlossenen 123 Industrieunternehmen eine stabile Auftragslage und Umsatzentwicklung in allen Schlüsselmarktsegmenten. Insbesondere im Bau- und baunahen Bereich zeigt sich eine positive Dynamik. Positiv entwickeln sich ebenfalls die Exporte von Klebstoffen.

Die Verfügbarkeit von Rohstoffen ist derzeit gut. Preisseitig verspürt die Klebstoffindustrie jedoch keine nennenswerte Entspannung. Da die Preisstellung für wichtige Schlüsselrohstoffe aktuell durch Angebot und Nachfrage und weniger durch Rohölpreise bzw. durch Spekulationen determiniert wird, fallen die Preissteigerungsraten vergleichsweise moderat aus. Die Kosten für die Mehrzahl der Vorprodukte zur Herstellung von Klebstoffen verharren allerdings weiterhin auf einem hohen Niveau, ebenso wie die Energie- und Transportkosten.

Die Umsatzzahlen des Statistischen Bundesamtes weisen für die deutsche Klebstoffindustrie im ersten Halbjahr 2012 ein Umsatzplus von 4 Prozent und für die ersten 7 Monate 2012 von 4,9 Prozent aus. Trotz der aktuell korrigierten Wirtschaftsprognosen und einer hohen Volatilität des wirtschaftlichen Umfeldes hält der Industrieverband Klebstoffe in diesem Jahr ein Gesamtbranchenwachstum in der Größenordnung von 3,5 Prozent auf insgesamt 3,4 Mrd. Euro für realistisch. Wenn sich die gesamtwirtschaftlichen Randbedingungen nicht dramatisch verändern, kann auch für das Jahr 2013 mit einem erneuten Wachstum von 3,5 Prozent gerechnet werden.
Damit wächst die deutsche Klebstoffindustrie weiterhin überproportional im Vergleich zur Gesamtwirtschaft. Dieses Plus an wirtschaftlichem Wachstum resultiert unmittelbar aus innovativen technologischen Entwicklungen, für die die Klebstoffindustrie als Systempartner praxisorientierte und wertschöpfende Lösungen anbietet.

\section{Neue Anwendungsfelder}

Es existiert bekanntlich kaum ein Anwendungsgebiet, in dem Klebstoffe ihre Leistungsfähigkeit nicht unter Beweis stellen können. Crash-optimierte Strukturklebstoffe im Automobilbau, biometrische Ausweise, Mobiltelefone, TabletPCs, Endoskope oder Windkraftanlagen sind nur einige Beispiele für Produkte, deren Realisierung nur durch den Einsatz innovativer und leistungsfähiger Klebstoffsysteme möglich geworden ist.

„Viele Marktnischen und technische Opportunitäten ergeben sich aus der extremen Anwendungsvielfalt von Klebstoffen, die sprichwörtlich von A bis Z, nämlich vom Automobil bis zur Zahnkrone reicht und die Basisexistenz für die etwa 125 im Klebstoffmarkt tätigen Unternehmen in Deutschland ist“, so Dr. Boris Tasche, Vorstandsvorsitzender des IVK.

Darüber hinaus erschließen sich $\mathrm{u}$. a. aus ordnungs- oder gesellschaftspolitischen Diskussionen und Zieldefinitionen neue Anwendungsfelder für Klebstoffe: Die derzeit intensiv geführte Diskussion zum Thema Nachhaltigkeit ist für die

„Viele Marktnischen und technische Opportunitäten ergeben sich aus der extremen Anwendungsvielfalt von Klebstoffen, die sprichwörtlich von A bis Z, nämlich vom Automobil bis zur Zahnkrone reicht und die Basisexistenz für die etwa 125 im Klebstoffmarkt tätigen Unternehmen in Deutschland ist", so Dr. Boris Tasche, Vorstandsvorsitzender des IVK. deutsche Klebstoffindustrie in verschiedener Hinsicht eine mehr als willkommene Gelegenheit, ihre Innovationskraft und ihr Leistungsprofil zu beweisen.

So lassen sich die Ziele zur Energieeinsparung im Wohnungsbau nur mit Klebstoffen realisieren - sowohl die Isolierung von Heizungs- oder Wasserrohren als auch die Installation von Wärmedämmverbundsystemen für Hausfassaden oder Dächer erfordern den Einsatz von Kleb- und Dichtstoffsystemen.

Des Weiteren wird die Nachhaltigkeitsdiskussion konsequenterweise auch zu Veränderungen von industriellen Fertigungsprozessen führen: Es müssen beispielsweise geeignete Alternativen zu hochenergetischen Verbindungstechnologien wie z. B. dem Schweißen oder Löten gefunden werden. Hierfür bietet die Klebstoffindustrie bereits heute innovative Hochleistungsklebstoffe an.

Last but not least ist der Schlüssel zur Herstellung ökoeffizienter Produkte der Leichtbau, der wiederum nur durch den Einsatz speziell entwickelter Klebstoffe möglich ist.

Weitere Infos: www.klebstoffe.com

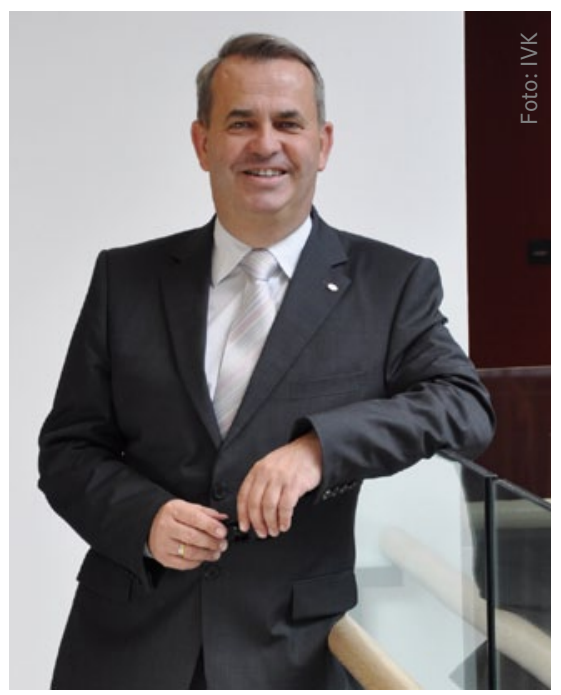

